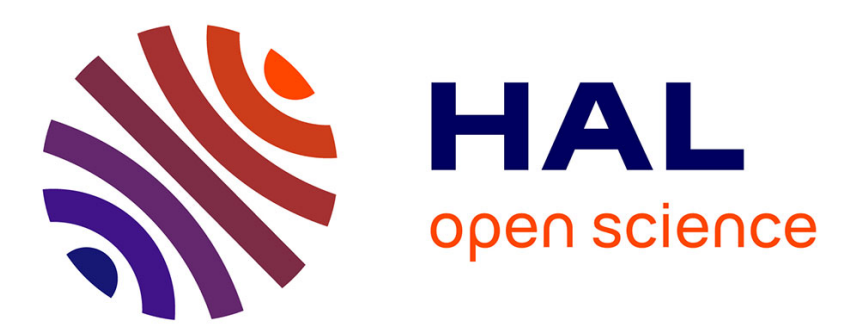

\title{
Fourier nanotransducers for phase-sensitive plasmonic biosensing
}

\author{
A. V. Kabashin, V. Kravets, F. Wu, V. O. Shipunova, S. M. Deyev, A. N. \\ Grigorenko
}

\section{- To cite this version:}

A. V. Kabashin, V. Kravets, F. Wu, V. O. Shipunova, S. M. Deyev, et al.. Fourier nanotransducers for phase-sensitive plasmonic biosensing. SPIE LASE, Feb 2020, San Francisco, United States. 10.1117/12.2551438 . hal-03100371

\section{HAL Id: hal-03100371 https://hal.science/hal-03100371}

Submitted on 6 Jan 2021

HAL is a multi-disciplinary open access archive for the deposit and dissemination of scientific research documents, whether they are published or not. The documents may come from teaching and research institutions in France or abroad, or from public or private research centers.
L'archive ouverte pluridisciplinaire HAL, est destinée au dépôt et à la diffusion de documents scientifiques de niveau recherche, publiés ou non, émanant des établissements d'enseignement et de recherche français ou étrangers, des laboratoires publics ou privés. 


\title{
Fourier nanotransducers for phase-sensitive plasmonic biosensing
}

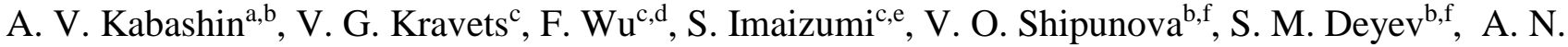 \\ Grigorenko ${ }^{\mathrm{b}}$
}

${ }^{a}$ Aix Marseille Univ, CNRS, LP3, Marseille, France, 13288; ${ }^{\mathrm{b}}$ MEPHI, Institute of Engineering Physics for Biomedicine, Kashirskoe sh. 31, 115409 Moscow; ' School of Physics and Astronomy, University of Manchester, Manchester, UK, M13 9PL ; ${ }^{\mathrm{d}}$ Key Laboratory for Non-Equilibrium Synthesis and Modulation of Condensed Matter (Ministry of Education), School of Science, Xi' an Jiaotong University, Xi'an, Shaanxi 710049, China; ${ }^{e}$ Key Fundamental Technology Research and Development Division 2, R\&D Center Sony Corporation, Atsugi Tec. 4-14-1 Asahi-cho, Atsugi 2430014, Japan; ${ }^{\mathrm{f}}$ Aix Shemyakin-Ovchinnikov Institute of Bioorganic Chemistry Russian Academy of Sciences, 16/10 Miklukho-Maklaya St, Moscow 117997, Russia

\begin{abstract}
We present phase-responding Fourier nanotransducers based on plasmonic metamaterials for ultrasensitive control of dynamic characteristics of 2D materials and functional biosensing interfaces. These nanotransducers are designed in such a way that they can confine light in $2 \mathrm{D}$ plane contacting with a probed ultrathin sample, gathering information about its properties, and then transmitting the information into discrete optical beams with amplified phase relations. To demonstrate their potential of Fourier transducers in biosensing, we designed Fourier nanotransducers based on periodic gold nanostructures and applied it in a newly developed protocol for the detection of important antibiotic chloramphenicol (CAP). Such biosensing tests showed the lower detection limit at $\mathrm{fg} \mathrm{mL}^{-1}$ level, which several orders of magnitude better than reported in the literature. The implementation of Fourier nanotransducers opens new opportunities for a radical improvement of current state-of-the art plasmonic biosensing technology.
\end{abstract}

Keywords: plasmonic biosensing, plasmonic metamaterials for biosensing, Fourier nanotransducers, Fourier metamaterials, plasmonic surface lattice resonances, diffractive coupling

\section{INTRODUCTION}

Relying on control of biomolecular binding events by refractive index (RI) monitoring, plasmonic biosensing has proved its efficiency and become a leading label-free technology for studies of biomolecular interactions on a gold-liquid interface [1-5]. Conventional plasmonic biosensors employ Surface Plasmon Resonance (SPR) phenomenon, which is produced with a help of 50-nm gold film in Kretschmann-Raether prism geometry [3]. More advanced plasmonic biosensors are based on localized plasmon resonances over gold nanostructures or nanoarrays [5]. Biomolecular interactions on gold lead to an increase of the thickness of a biomaterial layer, accompanied by the increase of refractive index of the medium contacting the sensor surface, which leads to a change of SPR or LPR coupling conditions. In this case, the course of biological interaction is typically monitored by following angular [1,2] or spectral [6] position of plasmonic resonant feature. The lower detection limit of SPR-based systems is of the order of $1 \mathrm{pg} / \mathrm{mm}^{2}$ of biomaterial accumulated on the biosensor surface [3,4]. LPR-based systems provide a higher detection limit of $\sim 1000 \mathrm{pg} / \mathrm{mm}^{2}[5]$, but they look much better compatible with current trends in biosensing focusing on the development of compact [7-10] or nanoscale [5, 11, 12] transducer implementations.

As we showed earlier [13-16], the employment of phase of light as a sensing parameter can provide a major breakthrough in sensitivity of plasmonic biosensors. Such a gain of sensitivity is due to nature of phase response: since the phase is not defined under zero value of system parameters, it can experience a jump in the minimum of resonance and the sharpness of this jump is inversely proportional to light intensity at the resonance $[15,16]$. The presence of such a jump of phase was observed in early experiments on SPR excitation [17] and then confirmed in many later studies. Profiting from phase sensitivity, a variety of schemes of phase detection based on interferometry, polarimetry and optical heterodyning have been proposed to enhance sensitivity of current state-of-the-art plasmonic biosensing (e.g., see [13-15, 20-26]), as well as 
to improve contrast of SPR images in conventional amplitude-sensitive schemes [27-29]. However, phase jumps are not very sharp under SPR as light intensity in the SPR dip cannot be lower than 9-10\% [16]. The limitation of natural plasmonic materials $\mathrm{Au}$ ) on the sharpness of the phase feature can be overcome by employing designed plasmonic metamaterials [30-33]. As an example, the employment of plasmonic metamaterials, enabling diffraction-coupled localized plasmon resonances, enables one to implement extreme Heaviside-like phase jumps, which open access to single molecule label-free detection [31]. We recently described several architectures of phase-sensitive plasmonic metamaterials, which are capable of providing ultrasensitive response from ultrathin films, including atomic layers of 2D materials and functional biosensing interfaces [34]. Termed as Fourier nanotransducers, such metamaterials make possible the concentration of electric field of light in 2D plane in order to sensitively accumulate information about its properties, and then encode the information into discrete optical beams with amplified phase relations to enable unlimited raw phase sensitivity to temporal variations in the examined object.

In this paper, we provide details of designs of Fourier nanotransducers and biosensing tests.

\section{RESULTS AND DISCUSSION}

\section{Definition of phase-responsive Fourier nanotransducers}

We define discrete Fourier materials (DFM) as periodically ordered materials, which can scatter light in the form of several reflected and diffracted beams described by a discrete sum of Fourier components [35]. Phase-responsive Fourier nanotransducers (PFN) are defined as discrete Fourier materials, which make possible concentration of light in 2D plane and thus enable abrupt dependence of the phase of the beam orders on properties of an adjacent 2D medium. The amplitude and the phase of any reflected or diffracted beams is encoded with information about the studied sample. We then postulate that phase of light produced by PFNs becomes an efficient parameter for characterization of 2D materials and interfaces if two conditions are satisfied:

(i) PFNs exhibit a sudden change of light beams in the discrete orders at the measurement conditions. For example, a loss of intensity in reflection is always accompanied by enhanced phase sensitivity $[15,16]$.

(ii) Probing light of PFN is confined in the plane of 2D material conditioning ultrasensitive phase response on variation of its properties. These two conditions set apart PFN materials from other patterned materials which can produce diffraction orders. The novelty of PFNs lies, therefore, in the purpose of metamaterial design: namely, PFNs are designed to generate amplified phase relations of reflected light necessary for sensing 2D materials and functional interfaces.

We can imagine several designs for the implementation of ultra-high phase response from 2D structures:

1. Implementation of darkness when the phase of the light changes in an abrupt fashion, while light is concentrated in $2 \mathrm{D}$ plane (Fig. 1a). Light darkness can be achieved using various effects: surface lattice plasmon resonances [36], beam interference [35], topological darkness [31], etc.) We showed in [16] that the increase of phase sensitivity over amplitude one, $G$, can be described by a simple relation $G \sim 1 / \Psi_{\min }$, where $\Psi_{\min } \approx E_{n} / E_{0}$.

2. Disappearance of the diffraction orders (Fig. 1b). The number of orders in DFM is not fixed and can change when the properties of a studied medium is changed. This would lead to a rearrangement of all beams produced by scattering and extremely fast changes of phase and amplitude of light in all orders. The most famous example of this case is Rayleigh cut-off phenomenon where a diffraction order disappears (or appears) for a sample fabricated on a substrate with refractive index different from that of the measured medium [37].

3. Employment of guided modes of light (Fig. 1c). This case does not require rearrangement of diffraction orders. Instead, a periodic arrangement of metamaterial elements produces optical beams channelled along the nanomaterials elements. As a result, these elements strongly modulate the phase of the channelled beam providing extremely high phase sensitivity to tiny changes of the medium surrounding nanomaterials. 


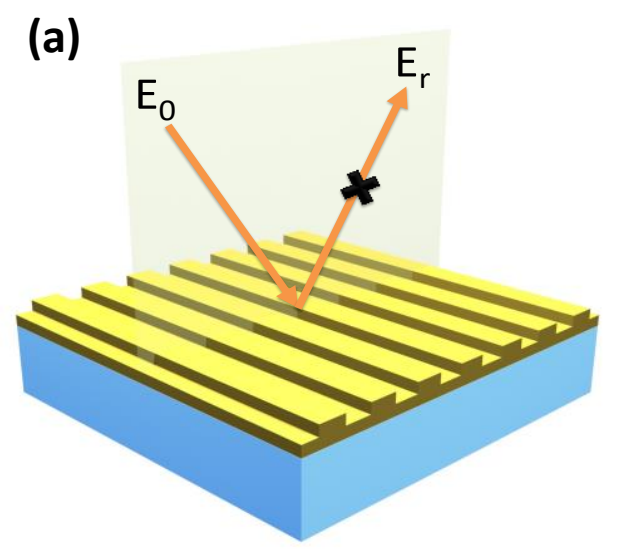

(b)

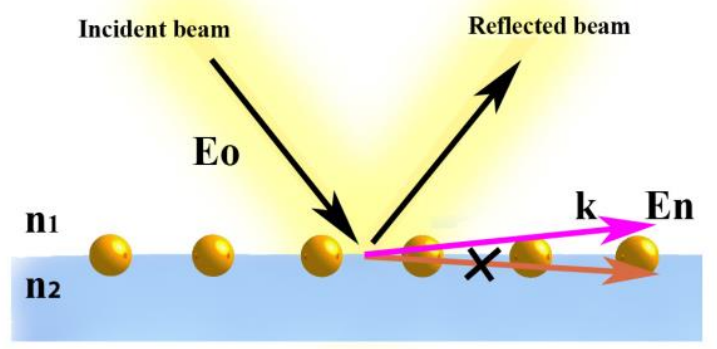

(c)

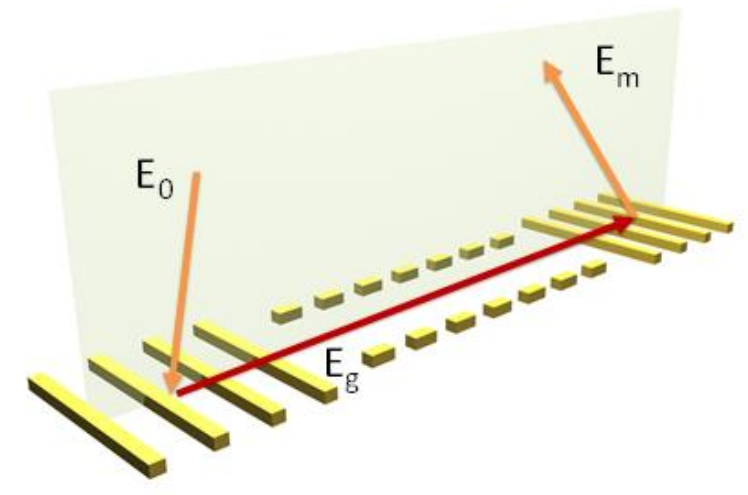

Figure 1. (a) PFNs enabling a drastic drop of intensity in reflected, transmitted or diffracted beams due to absorption in the system or other phenomena; (b) PFNs enabling the disappearance of a diffraction order (Rayleigh cut-off) or a redistribution of energy between them; (c) PFNs enabling light coupling to a guiding mode along nanomaterial elements.

\section{Diffraction edge phenomenon in fabricated PFMs}

For biosensing tests, we fabricated 2D nanotransducers on the basis of periodic arrays of double gold nanodots, which incorporate darkness, grazing beams and disappearance of diffraction orders in attenuated total reflection geometry. Simultaneous generation of these three phenomena becomes possible due to diffraction coupling of localized plasmon resonances over periodically arranged individual metamaterial elements (nanodots, nanostripes, etc.) [36]. Fig. 2a shows geometry of double nanodot arrays used in our experiments. The nanodots had the size of $150 \mathrm{~nm}$ (distance between dot centers $140 \mathrm{~nm}$ ), arranged in a periodic lattice (periodicity $320 \mathrm{~nm}$ ). Such arrays were produced by e-beam lithography (LEO-RAITH) on a clean microscopic glass substrate covered by a thin $\mathrm{Cr}$ sublayer. A double layered resist was used to improve lift-off (80nm of $495 \mathrm{kD}$ PMMA cast from a $3 \mathrm{wt} \%$ solution in anisole for the bottom resist layer and 50nm of 950kD PMMA cast from a $2 \mathrm{wt} \%$ solution in anisole for the top layer). After lithography we deposited $5 \mathrm{~nm}$ of $\mathrm{Cr}$ (to improve adhesion) and 80-90nm Au by electron beam evaporation which was followed by the lift-off procedure. The typical array size was $0.2 \times 0.2 \mathrm{~mm}^{2}$. The samples on a clean glass substrate were obtained from the samples fabricated on a $5 \mathrm{~nm} \mathrm{Cr}$ sublayer (routinely used to avoid charging during electron beam lithography) in which the $\mathrm{Cr}$ sublayer has been wet-etched after the fabrication procedure.

A scheme of such PFNs for examining 2D interfaces is shown in Figure 2b. One can see that the reflected beam shows the darkness at the wavelength of $\sim 600 \mathrm{~nm}$ realized due to diffraction coupling of localized plasmon resonances [36]. At the same time, the diffracted beam becomes really strong at the condition of Rayleigh anomaly [37] and disappears completely when the diffraction order moves into the substrate concentrating at the plane of the examined 2D object. The right inset in Figure 2 shows a well-defined spectral spike at the wavelength of the reflection darkness measured for grazing beam geometry, which confirms the generation of a planar mode at the diffraction edge conditions. 
(a)

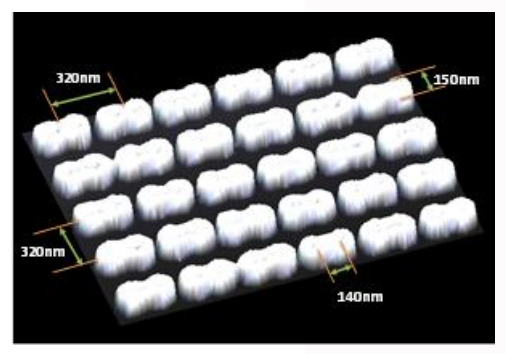

(b)
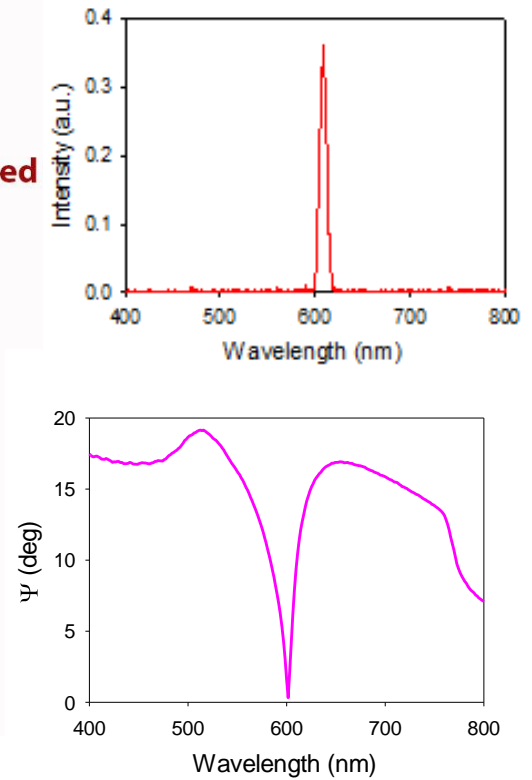

Figure 2. (a) Scanning Electron Microscopy image of 2D plasmonic array based on merged $150 \mathrm{~nm}$ nanoparticles used in our tests; (b) Diffraction edge phenomenon in fabricated PFM. The schematics of measurements are shown along with the reflection spectrum (bottom inset) and the grazing beam spectrum (right inset). The reflection spectrum was measured with the help of spectroscopic ellipsometer and is represented in ellipsometric reflection $\Psi$. The grazing spectrum was measured with the help of Ocean Optics spectrometer and is represented in arbitrary units.

\section{Biosensing experiment on quantification of antibiotic chloramphenicol (CAP)}

To assess the potential of PFNs in biosensing, we developed a novel protocol for a quantification of chloramphenicol (CAP, D(-)-threo-2-dichloroacetamido-1-p-nitro-phenyl-1,3-propanediol), a phenicol antibiotic with a broad spectrum of action directed against gram-positive and gram-negative bacteria, which prevents protein synthesis by inhibiting the peptidyl transferase activity of the bacterial ribosome. CAP is used in clinic for the ocular, ear and skin infections treatment as well as for veterinary medicine for treatment of some kinds of animal diseases. However, regular consumption of CAP (in particular, with food products) may lead to serious adverse effects for human health, such as leukemia, aplastic anemia and cardiovascular collapse Precise, sensitive and quantitative CAP detection both in fluids and solid samples has important clinical and social implications. A schematic description of functionalization steps on nanoparticle surface to implement the CAP detection protocol is given below.

A) Materials Proteins: Research Centre for Molecular Diagnostics and Therapy, Russia: Mouse anti-CAP IgG; Merck Millipore, Germany: Albumin from bovine serum, fraction V (BSA). Chemicals and other supplies: Sigma, Germany: 2(N-Morpholino)ethanesulfonic acid sodium salt (MES), Dimethyl sulfoxide (DMSO), N-(3-Dimethylaminopropyl)-N'ethylcarbodiimide hydrochloride (EDC), N-Hydroxysuccinimide (NHS); Pierce, USA: BCA Protein Assay Reagent (bicinchoninic acid); GE Healthcare Life Sciences, USA: NAP-5 columns; Sintez, Russia: Chloramphenicol succinate sodium salt (CAP); Dia-M, Russia: 1,4-Dithiothreitol (DTT); all other chemical reagents were of analytical grade and were used without further purification.

B) BSA labelling with CAP BSA labelled with chloramphenicol was prepared by carbodiimide conjugation of protein with chloramphenicol succinate sodium salt (CAP) as follows. CAP at $50 \mathrm{~g} / \mathrm{L}$ was incubated with EDC/NHS solution for 40 min at room temperature in solution of 50\% DMSO and 50\% 0.1 M MES buffer, pH 5.5 prior to addition to protein in borate buffer $\left(0.4 \mathrm{M} \mathrm{H}_{3} \mathrm{BO}_{3}, 70 \mathrm{mM} \mathrm{Na}_{2} \mathrm{~B}_{4} \mathrm{O}_{7} \cdot 10 \mathrm{H}_{2} \mathrm{O}, \mathrm{pH}\right.$ 8.0) for overnight incubation at room temperature. The proteins were labelled at concentration of $45 \mathrm{~g} / \mathrm{L}$ with molar ratio of BSA:CAP:EDC:NHS=1:40:80:80. Then protein was purified from reaction by-products using NAP-5 Columns (GE Healthcare Life Sciences, USA), and the concentration of conjugate was measured using BCA protein assay according to manufacturer's recommendations. 

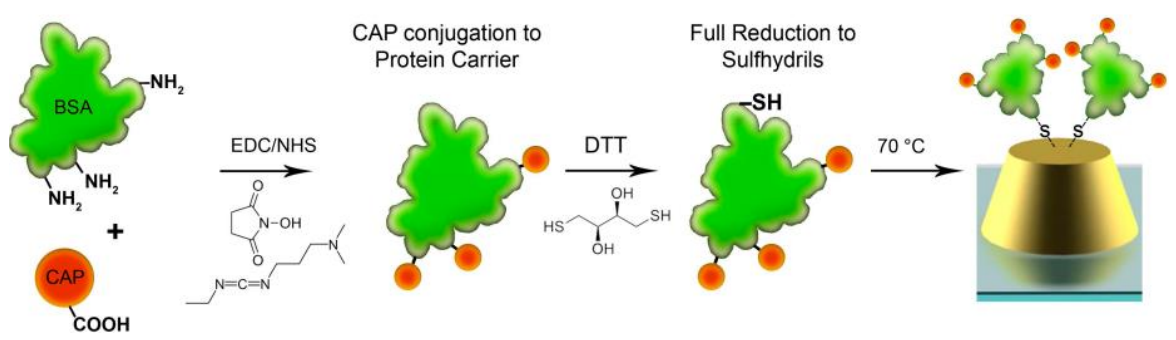

Figure 3. Functionalization of nanoparticle surface for enabling a protocol of chloramphenicol (CAP) detection. A carrier protein (bovine serum albumin, BSA) is first conjugated with CAP. BSA-CAP complex is then incubated with 1,4Dithiothreitol (DTT) in carbonate-bicarbonate buffer. Then, DTT-treated BSA-CAP complex is immobilized on gold nanoparticles.

C) Modification with proteins of glass slides coated with gold nanodots The surface of nanoparticles of the array was biofunctionalised with a carrier protein (bovine serum albumin, BSA) conjugated with CAP as schematically presented in Fig. 3. For nanoparticle modification, BSA-CAP (or BSA for control experiment) solution at $1 \mathrm{~g} / \mathrm{L}$ was incubated with DTT at molar ratio of BSA:DTT $=1: 34$ for $30 \mathrm{~min}$ at $70{ }^{\circ} \mathrm{C}$ in carbonate-bicarbonate buffer $\left(4 \mathrm{mM} \mathrm{Na}_{2} \mathrm{CO}_{3}, 50 \mathrm{mM}\right.$ $\mathrm{NaHCO}_{3}, \mathrm{pH}$ 9.2). Then, DTT-treated protein solution at $0.5 \mathrm{~g} / \mathrm{L}$ in carbonate-bicarbonate buffer was dropped onto the surface of nanoparticle array. After incubation, the surface was dried under air and rinsed with $\mathrm{dH}_{2} \mathrm{O}$ and phosphatebuffered saline (PBS, $137 \mathrm{mM} \mathrm{NaCl}, 2.7 \mathrm{mM} \mathrm{KCl}, 4.77 \mathrm{mM} \mathrm{Na}_{2} \mathrm{HPO}_{4} \cdot 2 \mathrm{H}_{2} \mathrm{O}, 1.7 \mathrm{mM} \mathrm{KH}_{2} \mathrm{PO}_{4}, \mathrm{pH} 7.4$ ).

D) CAP detection Samples under investigation containing different analyte concentrations were incubated with fixed concentration of anti-CAP antibodies. Namely, BSA-CAP covered nanoparticle array was sequentially exposed to 50 $\mu \mathrm{g} / \mathrm{mL}$ anti-CAP antibody in PBS with $1 \%$ BSA pre-incubated with sequentially 30 -fold decreasing concentrations of CAP (namely, its succinylated prodrug water-soluble form). The obtained complexes (CAP*anti-CAP IgG) were pumped through a liquid cell connected with the biofunctionalized nanoparticle array. The flow cell was pumped by the PBS buffer between different concentration points. In such an assay, when CAP concentration is high, all the antibodies are preblocked with analyte (CAP) and antibodies binding to the gold surface is impossible, thus, the detected signal is minimal (right panel of $4 \mathrm{a}$ ). Intermediate CAP concentration leads to the situation when significant part of antibody molecules in the sample under investigation are blocked with CAP (hence the procedure is termed competitive). In this case, the signal is directly proportional to the number of free antibody molecules, which are not bound with CAP in the solution (Fig. 4a, middle panel). In the case of minimum CAP concentration, a large amount of antibody molecules can bind to the surface of gold nanodots (Fig. 4a, left panel), thus providing a substantially large detected signal.

As shown in Figure 4b, the resonance gradually shifted and started to come to a saturation at concentrations of CAP lower than $10^{-3} \mathrm{ng} / \mathrm{mL}$. The detected signal forms a plateau when all the sites of binding on the surface of gold nanodots (namely, CAP molecules on the protein carrier BSA) are associated with antibodies and no signal increase is possible. It should be noted that the total range of spectral shift was not very large $(1.24 \mathrm{~nm})$, but our system could sensitively resolve the position of all points due to extremely small width of resonances (5-8 nm FWHM) and the application of statistical averaging methods to precisely measure the position of resonances. The recorded spectral shift was measured referring to that measured with the buffer solution alone (PBS with $1 \%$ BSA). The limit of detection (LOD) for minimal CAP concentration based on spectral data was determined by $3 \sigma$ criterion and found to be $25 \mathrm{pg} / \mathrm{mL}$, which is already better than LOD values from all previous studies for label-free methods (e.g., LOD equal to $0.26 \mathrm{ng} / \mathrm{mL}$ [38], $32.2 \mathrm{pg} / \mathrm{ml}$ [39], $30 \mathrm{pg} / \mathrm{mL}$ [40]) and not far from best values for label-based methods such as EIA assays ( $7.6 \mathrm{ng} / \mathrm{mL}$ [41]).

As shown in Figure 4b, phase started to evolve only after the third concentration of CAP, which was obviously related to the beginning of fast variation range within its singularity. Then, using phase as a signal parameter we could follow all subsequent concentrations of CAP, while the whole phase variation nearly reached its full dynamic range (180 deg). Finally, phase started to come to saturation at last points, which is consistent with complete binding of all active CAP sites on Au nanoparticles by the antibodies. The error of phase measurements provided by the ellipsometer for a fixed angle of incidence and the spectral operation range of $600-800 \mathrm{~nm}$ is $0.05 \mathrm{deg}$. Using this value for the error, we can estimate the relevant LOD as $27 \mathrm{fg} / \mathrm{mL}$, which is almost 3 orders of magnitude better than all values recorded by alternative techniques. As we reported in our previous study [15], a much better phase resolution of $10^{-3} \mathrm{deg}$. (and lower) can be obtained, e.g., by using advanced phase sensitive systems based on low-noisy acousto-optical modulators. It is easy to find that with such 
a phase resolution the LOD can be below $1 \mathrm{fg} / \mathrm{mL}$, i.e. in the sub-fg/mL level. It is important that this spectacular result can be obtained in the absence of the labelling step, which requires extra time and cost demands, as well as often occludes the binding site provoking false negative signals. Furthermore, the used optical arrangement enables one to follow biomolecular interactions in real time and thus obtain additional information on kinetic constants of reactions, which is hardly possible with labelling approaches.

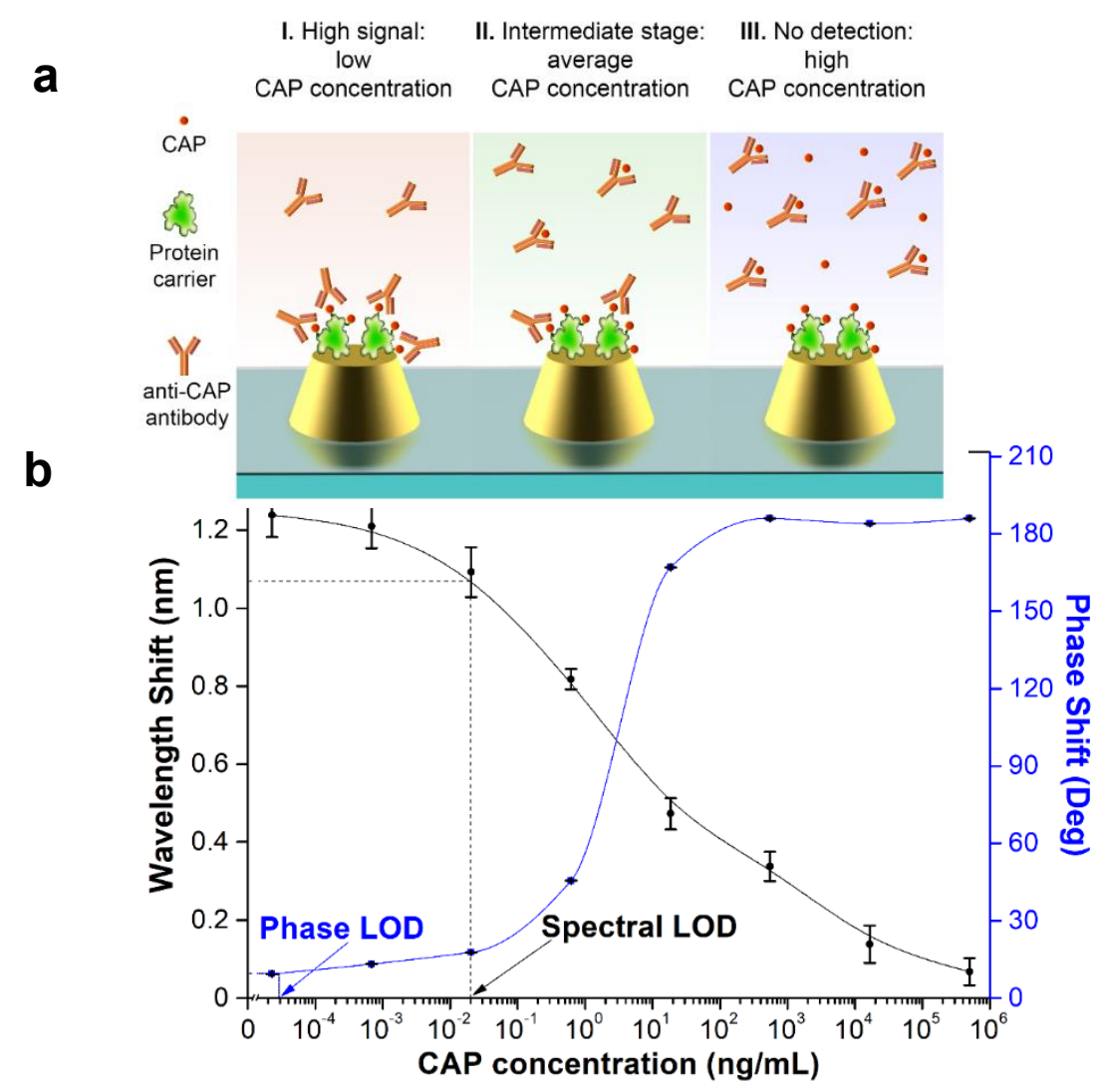

Figure 4. (a) In the competitive mode, CAP analyte in a sample under investigation is pre-incubated with anti-CAP antibody and the incubated complex (CAP*anti-CAP IgG) is pumped near CAP-coated Au nanoparticles. When CAP concentration is high, all antibodies are pre-blocked with CAP and their binding to surface-coated CAP is impossible (right panel). The decrease of CAP concentration leads to the appearance of free antibodies, which start to bind to CAP on the surface and the resulting signal is proportional to the number of surface-bound antibodies (middle panel). At low CAP concentrations (left panel) the recorded signal finally comes to a saturation corresponding to a complete binding of all sites on the surface of nanoparticles with antibodies (left panel); c) Dependence of spectral resonance minimum position (black) and light phase signal (blue) on concentration of CAP. The errors for spectral dependence were determined from the spectral resolution of the spectrometer $(\delta \lambda \cong 0.5 \mathrm{~nm})$ improved by fitting with the use of $N=7$ points to an experimental error of $\delta \lambda / \sqrt{N} \cong 0.2 \mathrm{~nm}$. The error was phase measurements was determined similarly and was about 0.05 Deg.

\section{CONCLUSION}

We presented Fourier nanotransducers, which make possible ultrasensitive control of dynamic properties of 2D materials and functional interfaces with unprecedented sensitivity. We showed that PFN can help to exceed LODs of current stateof-the-art label-free and label-based biosensors by more than 3 orders of magnitude, respectively. We believe that such hypersensitivity can radically advance label-free biosensing technology and eventually extend its application area beyond research laboratories. 


\section{ACKNOWLEDGEMENTS}

ANG acknowledges the support from the European Union's Horizon 2020 research and innovation programme under grant agreement No. 696656 GrapheneCore1/GrapheneCore2 and SONY research grant agreement. AVK acknowledges the support from Excellence Initiative of Aix-Marseille Univ - A*MIDEX, a French "Investissements d'Avenir" program. VOS acknowledges the support from the Russian Science Foundation, grant № 17-74-20146 (nanodots modification for biosensing experiment). AVK and SMD acknowledge support from Russian Science Foundation (Project 19-72-30012) for optical and chemical characterization of biosensor samples.

\section{REFERENCES}

[1] Liedberg, B., Nylander, C. and Lunström, I., "Surface plasmon resonance for gas detection and biosensing," Sensors and Actuators 4, 299-304 (1983).

[2] Liedberg, B., Nylander, C. and Lundström, I., "Biosensing with surface plasmon resonance - how it all started," Biosens. Bioelectron. 10(8), i-ix (1995).

[3] Homola, J. Surface Plasmon Resonance Based Sensors. (Springer, 2006).

[4] Tudos, A. J. and Schasfoort, R. B. M., "Handbook of Surface Plasmon Resonance" (2008).

[5] Anker, J. N., Hall, W. P., Lyandres, O., Shah, N. C., Zhao. J., Van Duyne, R. P. Biosensing with plasmonic nanosensors. Nature Mater 7, 442-453 (2006).

[6] Zhang, L.-M. and Uttamchandani, D., "Optical chemical sensing employing surface plasmon resonance," Electron. Lett. 24(23), 1469 (1988).

[7] Patskovsky, S., Kabashin, A. V, Meunier, M. and Luong, J. H. T., "Silicon-based surface plasmon resonance sensing with two surface plasmon polariton modes.," Appl. Opt. 42(34), 6905-6909 (2003).

[8] Piliarik, M., Homola, J., Maníková, Z. and Čtyroký, J., "Surface plasmon resonance sensor based on a singlemode polarization-maintaining optical fiber," Sensors Actuators, B Chem. 90(1-3), 236-242 (2003).

[9] Nemova, G., Kabashin, A. V. and Kashyap, R., "Surface plasmon-polariton Mach-Zehnder refractive index sensor," J. Opt. Soc. Am. B 25(10), 1673 (2008).

[10] Hassani, A., Gauvreau, B., Fehri, M. F., Kabashin, A. and Skorobogatiy, M., "Photonic crystal fiber and waveguide-based surface plasmon resonance sensors for application in the visible and near-IR," Electromagnetics 28(3), 198-213 (2008).

[11] Haes, A. J. and Van Duyne, R. P., "A nanoscale optical biosensor: sensitivity and selectivity of an approach based on the localized surface plasmon resonance spectroscopy of triangular silver nanoparticles.," J. Am. Chem. Soc. 124(35), 10596-10604 (2002).

[12] Kabashin, A. V, Evans, P., Pastkovsky, S., Hendren, W., Wurtz, G. A., Atkinson, R., Pollard, R., Podolskiy, V. A. and Zayats, A. V., "Plasmonic nanorod metamaterials for biosensing," Nat. Mater. 8(11), 867-871 (2009).

[13] Kabashin, A. V and Nikitin, P. I., "Interferometer based on a surface-plasmon resonance for sensor applications," Quantum Electron. 27(7), 653-654 (1997).

[14] Kabashin, A. V and Nikitin, P. I., "Surface plasmon resonance interferometer for bio-and chemical-sensors," Opt. Commun. 150 (1-6), 5-8 (1998).

[15] Grigorenko, A. N., Nikitin, P. I., Kabashin, A. V, "Phase Jumps and Interferometric Surface Plasmon Resonance Imaging,” Appl. Phys. Lett. 75, 3917-3919 (1999).

[16] Kabashin, A. V., Patskovsky, S., Grigorenko, A. N., Phase and amplitude sensitivities in Surface Plasmon Resonance bio- and chemical sensing. Opt. Express, 17, 21191-21204 (2009).

[17] Abeles, F. "Surface electromagnetic waves ellipsometry", Surf. Sci. 56, 237-251 (1976).

[18] Nelson, S. G., Johnston, K. S., Yee, S. S. "High sensitivity surface plasmon resonace sensor based on phase detection", Sens. Actuat. A 35-36, 187-191 (1996)

[19] Shen, S., Liu, T., Guo, J. "Optical Phase-Shift Detection of Surface Plasmon Resonance”, Appl. Opt. 37, 17471751 (1998).

[20] Notcovich, A. V. Zhuk, V., Lipson, S. G. "Surface plasmon resonance phase imaging, Appl. Phys. Lett. 76, 1665-1667 (2000).

[21] Ho, H.-P., Lam, W. W. "Application of differential phase measurement technique to surface plasmon resonance sensors", Sensor Actuat. B - Chem. 96, 554-559 (2003).

[22] Wu, S. Y., Ho, H.-P., Law, W. C., Lin, C., Kong, S. K. "Highly sensitive differential phase-sensitive surface plasmon resonance biosensor based on the Mach-Zehnder configuration," Opt. Lett. 29, 2378-2380 (2004). 
[23] Sheridan, A. k., Harris, R. D., Bartlett, P. N., Wilkinson, J. S. "Phase interrogation of an integrated optical SPR sensor, " Sensor Actuat. B - Chem. 97, 114-121 (2004).

[24] Naraoka, R. Kajikawa, K. "Phase detection of surface plasmon resonance using rotating analyzer method," Sensor Actuat. B - Chem. 107, 952-956 (2005).

[25] Law, W.-C., Markowicz, P., Yong, K.-T., Roy, I., Baev, A., Patskovsky, S., Kabashin, A. V., Ho, H.-P. and Prasad, P. N., "Wide dynamic range phase-sensitive surface plasmon resonance biosensor based on measuring the modulation harmonics," Biosens. Bioelectron. 23(5), 627-632 (2007).

[26] Huang, Y. H., Ho, H. P., Kong, S. K. and Kabashin, A. V., "Phase-sensitive surface plasmon resonance biosensors: Methodology, instrumentation and applications," Ann. Phys. 524(11), 637-662 (2012).

[27] Kabashin, A. V., Kochergin, V. E., Beloglazov, A. A. and Nikitin, P. I., "Phase-polarisation contrast for surface plasmon resonance biosensors," Biosens. Bioelectron. 13(12), 1263-1269 (1998).

[28] Kabashin, A. V., Kochergin, V. E. and Nikitin, P. I., "Surface plasmon resonance bio- and chemical sensors with phase-polarisation contrast," Sensor Actuat. B - Chem. 54(1), 51-56 (1999).

[29] Homola, J., Yee, S. S., "Novel polarization control scheme for spectral surface plasmon resonance sensors," Sensor Actuat. B - Chem, 1-3, 331-339 (1998)

[30] Kravets, V. G., Schedin, F., Kabashin, A. V and Grigorenko, A. N., "Sensitivity of collective plasmon modes of gold nanoresonators to local environment.," Opt. Lett. 35(7), 956-958 (2010).

[31] Kravets, V. G., Schedin, F., Jalil, R., Britnell, L., Gorbachev, R. V, Ansell, D., Thackray, B., Novoselov, K. S., Geim, a K., Kabashin, a V and Grigorenko, A. N., "Singular phase nano-optics in plasmonic metamaterials for label-free single-molecule detection," Nat. Mater. 12(4), 304-309 (2013).

[32] Aristov, A. I., Manousidaki, M., Danilov, A., Terzaki, K., Fotakis, C., Farsari, M., Kabashin, A. V. 3D plasmonic crystal metamaterials for ultra-sensitive biosensing. Sci. Rep., 6, 25380 (2016).

[33] Danilov, A., Tselikov, G., Wu, F., Kravets, V. G., Ozerov, I., Bedu, F., Grigorenko, A. N. and Kabashin, A. V., "Ultra-narrow surface lattice resonances in plasmonic metamaterial arrays for biosensing applications," Biosens. Bioelectron. 104, 102-112 (2018).

[34] Kabashin, A. V., Kravets, V. G., Wu, F., Imaizumi, S., Shipunova, V. O., Deyev, S. M., Grigorenko, A. N. "Phaseresponsive Fourier nano-transducers for probing 2D materials and functional interfaces", Adv. Func. Mater. 29, 1902692 (2019)

[35] Born, M., Wolf, E [Principles of Optics], Cambridge University Press, Cambridge (1999).

[36] Kravets, V. G., Kabashin, A. V., Barnes, W. L., Grigorenko, A. N. "Plasmonic Surface Lattice Resonances: A Review of Properties and Applications”, Chem. Rev., 118, 5912 (2018).

[37] Hessel, A., Oliner, A. A. “A new theory of Wood's anomalies on optical gratings,” Appl. Opt., 4, 1275 (1965).

[38] Fernández, F., Hegnerová, K., Piliarik, M., Sanchez-Baeza, F., Homola, J., Marco, M. P. “A label-free and portable multichannel surface plasmon resonance immunosensor for on site analysis of antibiotics in milk samples", Biosens. Bioelectron., 26, 1231 (2010).

[39] Yuan, J., Addo, J., Aguilar, M.-I., Wu, Y., "Surface plasmon resonance assay for chloramphenicol without surface regeneration", Anal. Biochem., 390, 97 (2009).

[40] Zhou, L., Gan, N., Zhou, Y., Li, T., Cao, Y., Chen, Y. "A label-free and universal platform for antibiotics detection based on microchip electrophoresis using aptamer probes", Talanta, 167, 544 (2017).

[41] Yu, X., He, Y., Jiang, J., Cui, H. A., “A competitive immunoassay for sensitive detection of small molecules chloramphenicol based on luminol functionalized silver nanoprobe”, Anal. Chim. Acta, 812, 236 (2014). 